\title{
MEAN-VALUE SURFACES
}

\section{MAXWELL READE AND E. F. BECKENBACH}

Introduction. The real functions

$$
x_{j}=x_{j}(u, v), \quad j=1,2,3,
$$

defined and continuous in a finite simply connected domain ${ }^{1} D$, will be said to define a surface $S$. If the first partial derivatives of the functions (1) are continuous in $D$, and if

$$
E(u, v)=G(u, v), \quad F(u, v)=0
$$

hold in $D$, where

$$
E(u, v) \equiv \sum_{j=1}^{3} x_{j u}^{2}, \quad F(u, v) \equiv \sum_{j=1}^{3} x_{j u} x_{j v}, \quad G(u, v) \equiv \sum_{j=1}^{3} x_{j v}^{2}
$$

are the coefficients of the first fundamental quadratic form of $S$, then the surface is said to be given in isothermic representation by the functions (1) and the parameters $u, v$ are said to be isothermic parameters; the map of $D$ on $S$ is conformal except where $E=G=0$.

In a previous paper, ${ }^{2}$ the authors studied the equation

$$
\sum_{j=1}^{3}\left[\int_{C} x_{j}(u, v) d z\right]^{2}=0, \quad z=u+i v,
$$

where $C$ is a circle in $D$; the following necessary and sufficient condition was obtained.

THEOREM A. If the functions (1) have continuous partial derivatives of the third order in a finite simply connected domain $D$, then a necessary and sufficient condition that they map $D$ isothermically either on a surface $S$ that lies on a sphere of finite non-null radius, such that circles are mapped on circles, or on a minimal surface $S$, is that (3) hold for each circle $C$ in $D$.

1. Mean-value surfaces. Let the coordinate functions (1) of a surface $S$ be continuous in a finite simply connected domain $D$; then the circular averages

1 A domain is a non-null connected open set.

${ }^{2}$ Generalizations to space of the Cauchy and Morera theorems, Transactions of this Society, vol. 49 (1941), pp. 354-377; in particular, see p. 365. 
(4) $x_{j}=A_{j, \rho}(u, v) \equiv \frac{1}{\pi \rho^{2}} \iint_{\xi^{2}+\eta^{2} \leqq \rho^{2}} x_{j}(u+\xi, v+\eta) d \xi d \eta, \quad j=1,2,3$,

where $\rho$ is a positive constant, will be said to define $a$ mean-value surface $S_{\rho}$ associated with $S$. We define

$$
A_{j, 0}(u, v) \equiv x_{j}(u, v), \quad j=1,2,3 .
$$

We note that the functions (4) are defined and have continuous partial derivatives of the first order in an open set of points $D_{\rho}$ which is interior to $D$; since $D_{\rho}$ is not necessarily a connected set, $S_{\rho}$ may consist of several pieces.

THEOREM 1.1. If the functions (1) are continuous in a simply connected domain $D$, then a necessary and sufficient condition that (3) hold for each circle $C$ in $D$ is that all mean-value surfaces $S_{\rho}$ associated with the surface $S$, defined by the functions (1), be given in isothermic representation by (4).

Proof. The first partial derivatives of the functions (4) are given by the relations ${ }^{3}$

$$
\begin{aligned}
& \frac{\partial A_{j, \rho}}{\partial u}=\frac{1}{\pi \rho^{2}} \int_{\xi^{2}+\eta^{2}=\rho^{2}} x_{j}(u+\xi, v+\eta) d \eta, \\
& \frac{\partial A_{j, \rho}}{\partial v}=-\frac{1}{\pi \rho^{2}} \int_{\xi^{2}+\eta^{2}=\rho^{2}} x_{j}(u+\xi, v+\eta) d \xi, \quad j=1,2,3,
\end{aligned}
$$

which are valid for points of $D_{\rho}$; hence

$$
\begin{aligned}
\sum_{j=1}^{3}\left[\int_{\xi^{2}+\eta_{2}=\rho^{2}} x_{j}(u+\xi, v+\eta)(d \xi+i d \eta)\right]^{2} & \\
& =-\pi^{2} \rho^{4}\left[E_{\rho}-G_{\rho}+2 i F_{\rho}\right\rfloor,
\end{aligned}
$$

where $E_{\rho}, F_{\rho}$ and $G_{\rho}$ are the coefficients of the first fundamental quadratic form of $S_{\rho}$. From (2), (3) and (5) we obtain the theorem.

From Theorems $\mathrm{A}$ and 1.1 we obtain the following result.

TheOREM 1.2. If the functions (1) have continuous partial derivatives of the third order in a finite simply connected domain $D$, then a necessary and sufficient condition that they map $D$ isothermically either on a surface $S$ that lies on a sphere of finite non-null radius, such that circles are mapped on circles, or on a minimal surface $S$, is that all mean-value surfaces $S_{\rho}$ associated with the surface $S$ defined by the functions (1) be given in isothermic representation by (4).

${ }^{3}$ T. Radó, Subharmonic Functions, Berlin, 1937, p. 11. 
2. Mean-value surfaces and transformations of axes. In $\$ 3$ we shall make use of the following observations.

2.1. $S_{\rho}$ is invariant under rigid transformations in the $\left(x_{1}, x_{2}, x_{3}\right)$ space; if

$$
x_{j}=a_{j}+\sum_{k=1}^{3} \lambda_{k j} x_{k}, \quad j=1,2,3,
$$

is a rigid transformation, then

$$
A_{j, \rho}^{\prime}(u, v)=a_{j}+\sum_{k=1}^{3} \lambda_{k j} A_{k, \rho}(u, v), \quad j=1,2,3,
$$

where

$$
A_{j, \rho}^{\prime}(u, v) \equiv \frac{1}{\pi \rho^{2}} \iint_{\xi^{2}+\eta^{2} \leqq \rho^{2}} x_{j}^{\prime}(u+\xi, v+\eta) d \xi d \eta, \quad j=1,2,3 .
$$

2.2. $S_{\rho}$ is invariant under each of the reflections

$$
u^{\prime}=u, v^{\prime}=-v,
$$

and

$$
u^{\prime}=-u, v^{\prime}=v
$$

If, for example,

$$
x_{j}^{\prime}\left(u^{\prime}, v^{\prime}\right) \equiv x_{j}\left(u^{\prime},-v^{\prime}\right), \quad j=1,2,3,
$$

then

$$
A_{j, \rho}^{\prime}\left(u^{\prime}, v^{\prime}\right)=A_{j, \rho}\left(u^{\prime},-v^{\prime}\right), \quad j=1,2,3,
$$

where

(6) $A_{j, \rho}^{\prime}\left(u^{\prime}, v^{\prime}\right) \equiv \frac{1}{\pi \rho^{2}} \iint_{\xi^{2}+\eta^{2} \leqq \rho^{2}} x_{j}^{\prime}\left(u^{\prime}+\xi, v^{\prime}+\eta\right) d \xi d \eta, \quad j=1,2,3$.

2.3. Similarly, $S_{\rho}$ is invariant under rigid transformations in the $(u, v)$-plane.

2.4. Under the transformation

$$
u^{\prime}=\alpha u, \quad v^{\prime}=\alpha v, \quad \alpha>0,
$$

the mean-value surface $S_{\rho}$ is transformed into the mean-value surface $S_{\alpha \rho}^{\prime}$ whose coordinate functions are given by $(6)$, where

$$
x_{j}^{\prime}\left(u^{\prime}, v^{\prime}\right) \equiv x_{j}\left(\frac{u^{\prime}}{\alpha}, \frac{v^{\prime}}{\alpha}\right), \quad j=1,2,3 .
$$


Hence for $\alpha$ fixed, the family of mean-value surfaces $\left[S_{\rho}\right]$ associated with a given surface $S$ is identical with the family of mean-value surfaces whose coordinate functions are given by (6); under the transformation (7), each member of one family is congruent to a member of the second family.

3. Conformal mean-value surfaces. Since, by Theorem 1.2, the only smooth surfaces in isothermic representation for which all associated mean-value surfaces are given in isothermic representation by (4) are (a) spherical surfaces, in representation whereby circles are mapped on circles, and (b) minimal surfaces, the question arises as to the nature of the mean-value surfaces in these two cases.

THEOREM 3.1. If the functions (1) map a finite simply connected domain $D$ isothermically on a minimal surface $S$, then each mean-value surface $S_{\rho}$ associated with $S$ is a minimal surface given in isothermic representation by (4) and coinciding with $S$ for $(u, v)$ in $D_{\rho}$.

Proof. By a theorem of Weierstrass, ${ }^{4}$ the functions (1) are harmonic in $D$; consequently it follows from the mean-value property of harmonic functions that the functions (4) coincide with the functions (1) in the open set $D_{\rho}$. Hence all mean-value surfaces associated with minimal surfaces given in isothermic representation by (1) are themselves minimal surfaces given in isothermic representation by (4); the surface $S_{\rho}$ coincides with $S$ for $(u, v)$ in $D_{\rho}$.

THEOREM 3.2. If the functions (1) are not identically constant and if they map a finite simply connected domain $D$ isothermically on a surface $S$ that lies on a sphere $S$ of finite non-null radius a, such that circles are mapped on circles, then each mean-value surface $S_{\rho}$ associated with $S$ lies on a surface of revolution $T_{\rho}$ and is given in isothermic representation by (4). Further, for $0<\rho<\infty, T_{\rho}$ is not a sphere.

PRoof. It has been pointed out, in a recently published paper, ${ }^{5}$ that the functions (1) may be continued isothermically to map the entire closed $u, v$-plane isothermically on the whole of $\mathcal{S}$; further, the functions (1) have the representation

${ }^{4}$ If the functions (1) are harmonic in $D$, and if (2) holds in $D$, then the functions (1) are said to form a triple of conjugate harmonic functions; see E. F. Beckenbach and T. Rad6, Subharmonic functions and minimal surfaces, Transactions of this Society, vol. 35 (1933), pp. 648-661. Then the theorem of Weierstrass may be stated as follows. A necessary and sufficient condition that the functions (1), defined in the domain $D$, be the coordinate functions of a minimal surface given in isothermic representation is that they form a triple of conjugate harmonic functions; loc. cit., p. 649.

${ }^{5}$ Loc. cit., see Footnote 2; see p. 375. 


$$
\begin{aligned}
& x_{1}=x_{1}(u, v) \equiv a_{1}+\frac{2 a|k| R f(z)}{|f(z)|^{2}+k^{2}}, \\
& x_{2}=x_{2}(u, v) \equiv a_{2}+\frac{2 a|k| \jmath f(z)}{|f(z)|^{2}+k^{2}}, \\
& x_{3}=x_{3}(u, v) \equiv k\left[1-\frac{2 a|k|}{|f(z)|^{2}+k^{2}}\right],
\end{aligned}
$$

where $f(z)$ has one of the following forms:

$$
f(z) \equiv \alpha z+\beta, \quad f(z) \equiv \alpha \bar{z}+\beta,
$$

where $\alpha$ and $\beta$ are constants. In (8), $k=a_{3} \pm a,|k|$ is the maximum of the two quantities $\left|a_{3}+a\right|$ and $\left|a_{3}-a\right|$ and $\left(a_{1}, a_{2}, a_{3}\right)$ are the coordinates of the center of $\mathcal{S}$.

From 2.1-2.3, it follows that we may assume $a_{1}=a_{2}=a_{3}=0, k=a$, in (8), and that we may assume the function $f(z)$ is given by

$$
f(z) \equiv \alpha z, \quad \alpha>0 .
$$

This is equivalent to assuming that the point $z=\infty$ corresponds to the point $P:(0,0, a)$ on $\mathcal{S}$, that the point $z=0$ corresponds to the point $P^{\prime}:(0,0,-a)$ which is diametrically opposite $P$, and that the point in the $z$-plane corresponding to $(a, 0,0)$ is real and positive. Since we are investigating all mean-value surfaces associated with $S$, it follows from 2.4 that we may take $\alpha=1$ in (9), in which case the functions (1) have the following familiar representation, as given by (8):

$$
\begin{aligned}
& x_{1}=x_{1}(u, v) \equiv \frac{2 a^{2} u}{u^{2}+v^{2}+a^{2}}, \\
& x_{2}=x_{2}(u, v) \equiv \frac{2 a^{2} v}{u^{2}+v^{2}+a^{2}}, \\
& x_{3}=x_{3}(u, v) \equiv a\left[1-\frac{2}{u^{2}+v^{2}+a^{2}}\right] .
\end{aligned}
$$

If $C_{r}$ is the circle $u^{2}+v^{2}=r^{2}$, and if

then (4) and (10) yield

$$
z=u+i v=r e^{i \theta},
$$

$$
\begin{aligned}
A_{1, \rho}(u, v)+i A_{2, \rho}(u, v) & =e^{i \theta}\left[A_{1, \rho}(r, 0)+i A_{2, \rho}(r, 0)\right], \\
A_{3, \rho}(u, v) & =A_{3, \rho}(r, 0),
\end{aligned}
$$


from which it follows that the map of $C_{r}$ on the mean-value surface $T_{\rho}$, associated with the sphere $\mathcal{S}$ which is defined by $(10)$, is a circle $C_{r}^{*}$ in a plane perpendicular to the $x_{3}$-axis; moreover, the center of $C_{r}^{*}$ is on the $x_{3}$-axis. Since $S_{\rho}$ lies on $T_{\rho}$, it follows that the functions (4) map $D_{\rho}$ isothermically on a surface that lies on the surface of revolution $T_{\rho}$.

In $\$ 4$ it will appear that for $0<\rho<\infty, T_{\rho}$ is not a sphere. Nevertheless, we shall call $T_{\rho}$ a mean-value sphere.

4. Mean-value spheres. The mean-value sphere $T_{\rho}$ is a surface of revolution about the $x_{3}$-axis. Accordingly, to investigate $T_{\rho}$, it is sufficient to study the intersection $T_{\rho}^{*}$ of $T_{\rho}$ with the plane $x_{2}=0$. Since, by (10),

$$
x_{2}(u,-v) \equiv-x_{2}(u, v),
$$

it follows that the intersection of $T_{\rho}$ with the plane $x_{2}=0$ can be obtained from (4) by setting $v=0$ in (10). A computation yields the following coordinate functions for $T_{\rho}^{*}$ :

$$
\begin{aligned}
x_{1} & =A_{1, \rho}(u, 0) \\
& \equiv 4 a^{2} u /\left[a^{2}+\rho^{2}+u^{2}+\left(\left(a^{2}+\rho^{2}-u^{2}\right)^{2}+4 a^{2} u^{2}\right)^{1 / 2}\right], \\
x_{3} & =A_{3, \rho}(u, 0) \\
& \equiv a-\frac{2 a^{3}}{\rho^{2}} \log \frac{a^{2}+\rho^{2}-u^{2}+\left(\left(a^{2}+\rho^{2}-u^{2}\right)^{2}+4 a^{2} u^{2}\right)^{1 / 2}}{2 a^{2}} .
\end{aligned}
$$

We make the following observations.

4.1. The curve $T^{*}$ is symmetric about the $x_{3}$-axis; since

$$
\begin{aligned}
& A_{1, \rho}(u, 0) \equiv A_{1, \rho}\left(\frac{u^{2}+\rho^{2}}{u}, 0\right) \\
& A_{3, \rho}(u, 0) \equiv 2 a-\frac{2 a^{3}}{\rho^{2}} \log \frac{a^{2}+\rho^{2}}{a^{2}}-A_{3, \rho}\left(\frac{u^{2}+\rho^{2}}{u}, 0\right),
\end{aligned}
$$

it follows that $T_{\rho}^{*}$ is also symmetric about the line

$$
x_{3}=A_{3, \rho}\left(\left(a^{2}+\rho^{2}\right)^{1 / 2}, 0\right)=a-\frac{a^{3}}{\rho^{2}} \log \frac{a^{2}+\rho^{2}}{a^{2}} .
$$

From this symmetry and from the relations,

$$
m=\frac{2 a u}{a^{2}+\rho^{2}-u^{2}}, \quad \frac{d m}{d u}=\frac{2 a\left(a^{2}+\rho^{2}+u^{2}\right)}{\left(a^{2}+\rho^{2}-u^{2}\right)^{2}},
$$

satisfied by the slope $m$, it follows that $T_{\rho}^{*}$ is convex. 
4.2. The height $h$ and the width $l$ of $T_{\rho}^{*}$ are given by

$$
h=\frac{2 a^{3}}{\rho^{2}} \log \frac{a^{2}+\rho^{2}}{a^{2}}, \quad l=\frac{4 a^{2}}{\left(a^{2}+\rho^{2}\right)^{1 / 2}+a} ;
$$

hence for $0<\rho<\infty$ the curve $T_{\rho}^{*}$ is not a circle. Moreover, since

$$
\lim _{\rho \rightarrow \infty} \frac{l}{h}=\infty
$$

it follows that $T_{\rho}^{*}$ "flattens out" while approaching the point $(0, a)$, as $\rho \rightarrow \infty$.

4.3. Each member of the family $\left[T_{\rho}^{*}\right]$ passes through the point $(0, a)$ and is tangent there to every other member of the family. For $\rho=0, T_{\rho}^{*}$ is the circle $x_{1}^{2}+x_{3}^{2}=a^{2}$, and for $\rho=\infty, T_{\rho}^{*}$ is the point $(0, a)$.

4.4. A computation shows that for $0 \leqq \rho<\rho^{\prime}, T_{\rho^{\prime}}{ }^{*}$ is inside $T_{\rho}^{*}$, except for their common point of tangency.

The figure shows $T_{\rho}^{*}$ for $\rho=0,1,2(6)^{1 / 2}, \infty ; a=1$.

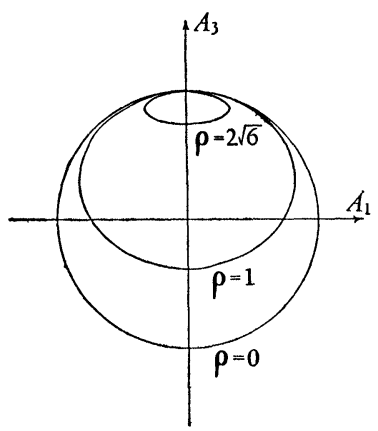

From 4.1-4.4 it follows that the family of surfaces $\left[T_{\rho}\right]$ consists of convex surfaces each of which passes through the point $(0,0, a)$ in $\left(x_{1}, x_{2}, x_{3}\right)$-space and is tangent there to every other member of the family. The surface $T_{\rho}$ is a surface of revolution about the $x_{3}$-axis and is symmetric with respect to the plane

$$
x_{3}=a-\frac{a^{3}}{\rho^{2}} \log \frac{a^{2}+\rho^{2}}{a^{2}} .
$$

Since the ratio of width to height $\rightarrow \infty$ as $\rho \rightarrow \infty$, it follows that $T_{\rho}$ "flattens out" as $\rho \rightarrow \infty$. For $\rho=0, T_{\rho}$ is the sphere about the origin with radius $a$, and for $\rho=\infty, T_{\rho}$ is the point $(0,0, a)$. For $0 \leqq \rho^{\prime}<\rho$, $T_{\rho}$ is inside $T_{\rho^{\prime}}$, except for their common point of tangency. From (11) we obtain the following isothermic representation for $T_{\rho}$ : 


$$
\begin{aligned}
x_{1} & =A_{1, \rho}(u, v) \\
& \equiv \frac{a^{2} u}{\rho^{2}\left(u^{2}+v^{2}\right)}\left[a^{2}+u^{2}+v^{2}+\rho^{2}-\left(\left(a^{2}+\rho^{2}-u^{2}-v^{2}\right)^{2}+4 a^{2}\left(u^{2}+v^{2}\right)\right)^{1 / 2}\right], \\
x_{2} & =A_{2, \rho}(u, v) \\
& \equiv \frac{a^{2} v}{\rho^{2}\left(u^{2}+v^{2}\right)}\left[a^{2}+u^{2}+v^{2}+\rho^{2}-\left(\left(a^{2}+\rho^{2}-u^{2}-v^{2}\right)^{2}+4 a^{2}\left(u^{2}+v^{2}\right)\right)^{1 / 2}\right], \\
x_{3} & =A_{3, \rho}(u, v) \\
& \equiv a-\frac{2 a^{3}}{\rho^{2}} \log \left[\frac{a^{2}-u^{2}-v^{2}+\rho^{2}+\left(\left(a^{2}+\rho^{2}-u^{2}-v^{2}\right)^{2}+4 a^{2}\left(u^{2}+v^{2}\right)\right)^{1 / 2}}{2 a^{2}}\right] .
\end{aligned}
$$

The Ohio State University and

The University of Michigan

\section{NOTE ON THE DISTRIBUTION OF VALUES OF THE ARITHMETIC FUNCTION $d(m)^{1}$}

M. KAC

1. Introduction. Recently Dr. Erdös and the present writer ${ }^{2}$ proved the following theorem:

If $\nu(m)$ denotes the number of different prime divisors of $m$ and $k_{n}(\omega)$ the number of positive integers $m \leqq n$ for which

$$
\nu(m) \leqq \lg \lg n+\omega(2 \lg \lg n)^{1 / 2},
$$

then

$$
\lim _{n \rightarrow \infty} \frac{k_{n}(\omega)}{n}=\pi^{-1 / 2} \int_{-\infty}^{\omega} e^{-u^{2}} d u=D(\omega) .
$$

The purpose of this note is to derive a similar theorem concerning the function $d(m)$ which denotes the number of all different divisors of $m$ ( 1 and $m$ are included).

In fact we are going to prove the following theorem:

If $r_{n}(\omega)$ denotes the number of positive integers $m \leqq n$ for which

$$
d(m) \leqq 2^{\lg \lg n+\omega(2 \lg \lg n)^{1 / 2}},
$$

1 Presented to the Society, May 2, 1941.

2 P. Erdös and M. Kac, The Gaussian law of errors in the theory of additive number theoretic functions, American Journal of Mathematics, vol. 62, pp. 738-742. 